\title{
What Makes Consumer Perception of Online Review Helpfulness: Synthesizing the Past to Guide Future Research
}

\author{
Wenjie Fan \\ Aalto University School of Business \\ wenjie.fan@aalto.fi
}

\begin{abstract}
A growing body of academic research has aimed to investigate the helpfulness of online customer reviews (OCRs) given their prevalence and the need to better understand their appraisal mechanisms. However, past studies have applied varied methods and reported conflicting findings. This study aims to improve the understanding of the contributors to OCR helpfulness by synthesizing past studies on the topic. Based on a systematic literature review, a summary of the precursors to OCR helpfulness is provided. We decipher both the consistent and conflicting results and discuss the possible explanations for these mixed findings. By summarizing past studies, the review also points out possible directions for future research.
\end{abstract}

\section{Introduction}

In today's digital age, consumers increasingly rely on online customer reviews (OCRs) before buying a product in order to reduce information asymmetry and justify their purchase decisions $[1,2]$. Over 90 percent of consumers admit that OCRs influence their purchasing decisions [3]. Indeed, by viewing the OCRs of those who have purchased and used a product, consumers can reduce the perceived uncertainty and risk associated with purchasing a product [4]. OCRs have gained importance due to their non-commercial character, richness of their content, and impact on consumers' decision making.

OCRs, originally introduced to reduce consumers' search costs, have themselves become a source of information overload. The significant growth of online platforms and communities has provided ample channels for OCR generation and dissemination and increased their influence in various markets. However, not all OCRs are equally informative and useful [5]. Moreover, the sheer volume of OCRs available to consumers may cause information overload $[1,6,7,8]$, thus exhausting consumers trying to identify highquality reviews to support their decision making.

To address this problem, OCR platforms enable users to indicate OCR informativeness by voting reviews as helpful or unhelpful [9]. Helpful OCRs have become an important instrument in customers' decision making [10]. To better understand this appraisal mechanism, ample studies have been performed in the past decade to attempt to identify the most influential contributors to OCR helpfulness. However, inconsistent results have been reported, and studies have adopted varied research methods and analyzed distinct datasets. For instance, the effect of star rating on review helpfulness is not clear. Some studies suggest negative reviews are perceived as more helpful under certain circumstances $[11,12]$, whereas the opposite findings were obtained in others $[5,9,13]$. Although empirical evidence shows objective reviews are preferred by consumers [14, 15], Scholz and Dorner [13] claimed that objective reviews are more helpful for search goods, whereas subjective reviews are more helpful for experience goods. Without systematically synthesizing the critical contributors to OCR helpfulness, it is difficult to fully comprehend the findings of the existing literature.

It is also relevant that OCRs are often unstructured and primarily qualitative in nature [10]. However, advanced techniques to understand OCR content are available, and the OCR literature continues to evolve and identify new factors affecting OCR helpfulness. For instance, improvements in natural language processing (NLP) and text mining techniques have enabled researchers with the ability to extract more characteristics embedded in review texts, such as sidedness [16, 17], emotions [6, 18], linguistic features $[14,15]$, and so forth. Previous literature reviews have not covered all of these newly emerging attributes [19, 20]. Moreover, previous reviews have only summarized the conflicting findings without discussing the inconsistencies of the results or how to improve the utilized models. This study aims to fill in these gaps by producing a systematic summary of the most recent publications on OCR helpfulness to provide researchers with up to date status of the literature and pointing out directions for future research.

At the same time, this article discusses the different contributors to OCR helpfulness and aims to decipher 
the mixed findings reported in the existing literature, with the objective of better understanding their nature and the underlying appraisal mechanisms operating on online platforms. In particular, to provide researchers and practitioners with insight into ORC helpfulness, this study systematically reviews, analyzes, and synthesizes the factors affecting consumers' perceptions of OCR helpfulness. In line with the methodological guidance provided by Webster and Watson [21], 68 articles published between 2007 and 2020 were analyzed in depth.

In addition, this study contributes to both theory and practice alike. It contributes to the growing literature on OCRs by creating a theoretical framework to facilitate understanding consumer perception of OCR helpfulness. Furthermore, through the analysis of review-, reviewer-, and product-related contributors, we offer a summary of the consistent and conflicting findings and discuss why inconsistent results exist or how to improve the research. For the presentation of our findings, we rely on trend map visualization of the contributors to OCR helpfulness in order to provide a holistic and straightforward overview of the factors addressed in the existing literature.

The remaining part of this paper is organized as follows: In the next section, we outline the adopted research methodology. Then, we present the findings of our literature analysis, covering both consistent and inconsistent findings. We elaborate the possible explanations for these inconclusive findings and highlight the emerging trends. The final sections provide a discussion of the implications and conclusion, and in addition guidance for future research.

\section{Literature identification and analysis}

Following the structured approach recommended by Webster and Watson [21], which minimizes bias and error and adds rigor to the literature review process, this work conducts a systematic literature review of the existing research on OCR helpfulness. Since the objectives are to summarize the current research on the contributors to consumer perception of OCR helpfulness and to provide guidance for further research in this field, we traverse and analyze the OCR helpfulness literature to provide a comprehensive overview of the findings and discussion.

In the literature search stage, we used two methods to identify relevant articles in the literature. First, we conducted a systematic search using a number of scientific research databases to retrieve data and construct the review sample. To ensure the comprehensiveness of the coverage of OCR helpfulness articles in our review, we conducted manual keyword searches on Science Direct, EBSCOHost, ProQuest, and Google Scholar, which are the four largest and widely used research databases and search engines for academic research [22]. In particular, we used the keywords "helpfulness/usefulness/trustworthiness," "online (customer/consumer) review," "word of mouth," "WOM," and "eWOM" to search for related articles. Second, the references of encountered articles were traced to guarantee the extensiveness of our literature database. The combination of these two literature search methods ensured that no major OCR helpfulness articles were ignored.

According to the systematic review methodology [21], inclusion and exclusion criteria were applied to the preliminary sample of articles. These efforts ensured that the review sample was appropriate for the current literature analysis. The criteria for practical screening for inclusion were as follows: (1) The paper must be academic and published in a peer reviewed journal. (2) OCR helpfulness must be the main focus of the paper. Because our objective focused on review-, reviewerand product-related factors contributing to OCR helpfulness, we filtered out articles that solely focused on predicting OCR helpfulness or developing natural language processing and text classification methods. After this stage, the extraction stage consisted in both quality appraisal and applicable information extraction [21]. By downloading and reading the abstracts of the articles in the initial dataset, we ascertained that for any paper in our final collection, it focused primarily on the drivers of OCR helpfulness. Specifically, to be assured of the comprehensiveness of our review, we kept both empirical research papers and those conducting experimental studies. Consequently, all the articles that should be collected in our literature review have been identified. By this process, we identified 68 relevant journal articles published between 2007 and 2020 and included them in our final sample.

We then extracted relevant information from each article in the review sample, including research method, theoretical grounds, studied factors, operationalization of the contributor(s), and findings. To provide a clear overview of the factors mentioned in the literature, a trend map of contributors to OCR helpfulness was developed (see Figure 1). This visualization makes it easier for readers to track shifts in the research of drivers of OCR helpfulness and, more importantly, their relationship. Over time, research efforts have been increasingly devoted to OCR helpfulness. Also, this trend map illustrates the time at which different OCR helpfulness contributors are initially investigated. The key findings are reported in the subsequent sections where we synthesize the findings to provide a comprehensive picture of the existing literature. 


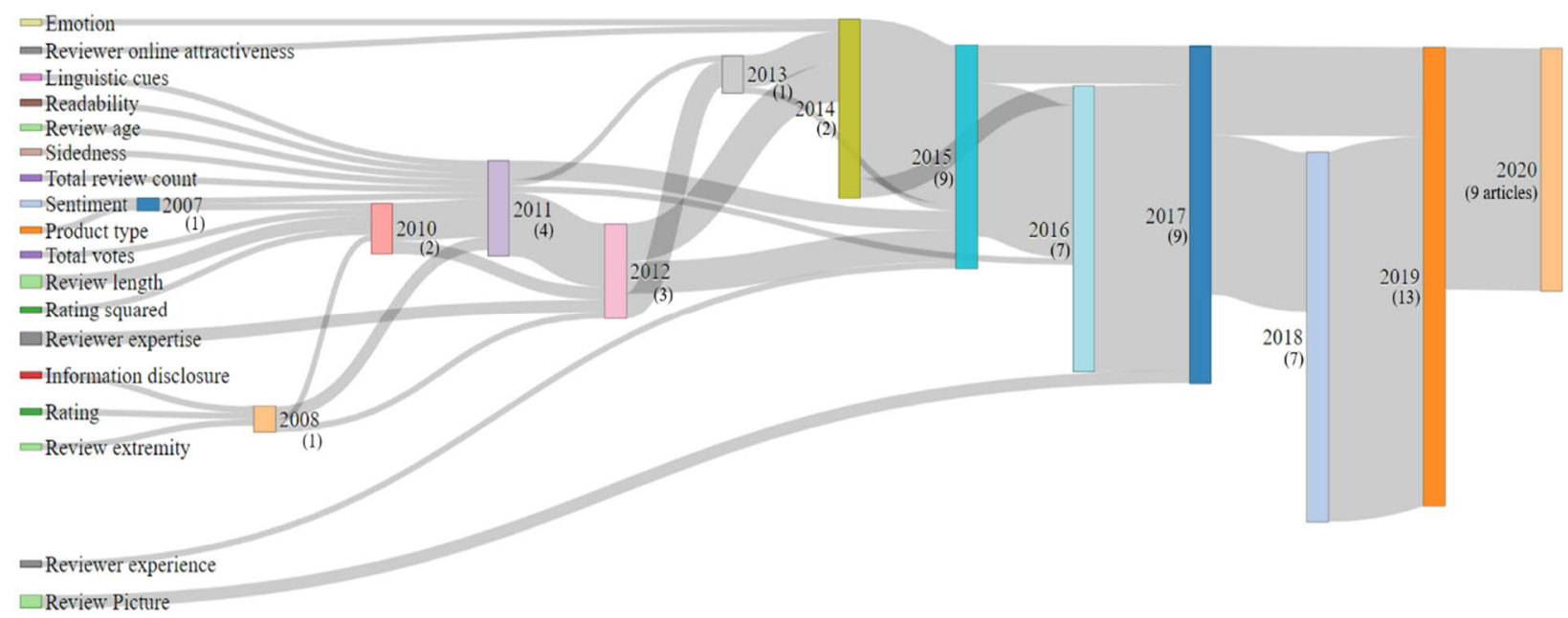

Figure 1 Trend map of contributors to OCR helpfulness

\section{Review of study findings}

The 68 papers that comprise our sample identified 18 contributors to OCR helpfulness (see Table 1). These precursors that have been extensively investigated in the past studies can be classified into three categories: 1) review-related characteristics (e.g., star rating, review length, review readability, and so forth), 2) productrelated characteristics (e.g., product type indicating experience goods or search goods [9], and total review number), and 3) reviewer-related characteristics (e.g., information disclosure, reviewer expertise, etc.). The definition and operationalization of the abovementioned factors, as well as their impacts on OCR helpfulness, are consolidated and listed in Table 1. In addition, both the consistent and the conflicting findings are presented and summarized in the remainder of this section.

Figure 2 presents the framework of OCR helpfulness: Review-related characteristics may increase or decrease the consumer perception of OCR helpfulness. Reviewer-related characteristics can increase the perceived OCR helpfulness. Finally, product-related characteristics can play a moderating role in this relationship between the first two categories and OCR helpfulness.

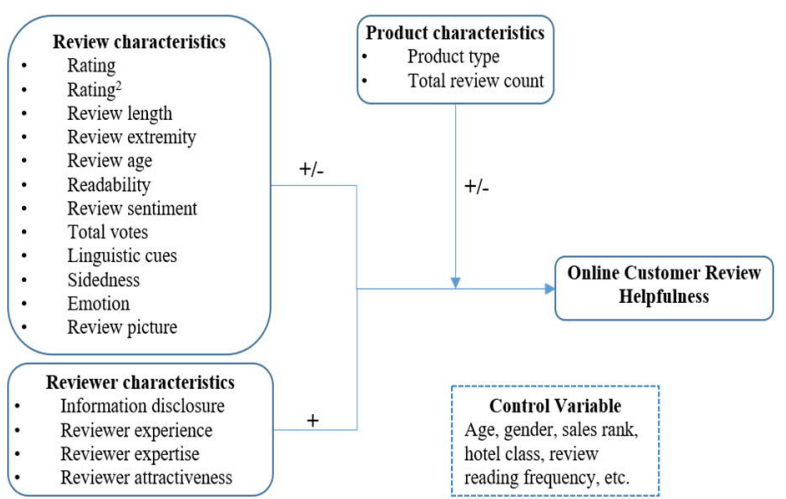

Figure 2 Framework of OCR helpfulness

\subsection{Consistent Findings}

Three out of the fourteen review and product characteristics, namely review length, readability, and review picture, were consistently reported to have positive effects on the perceived helpfulness of OCRs.

Table 1 Major contributors to OCR helpfulness

\begin{tabular}{|c|c|c|c|}
\hline Category & Contributor & Operationalization & Studies and effects \\
\hline \multirow{5}{*}{ 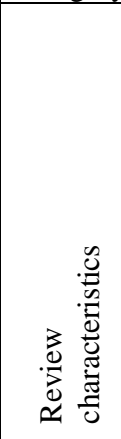 } & \multirow{3}{*}{ Rating } & \multirow{3}{*}{ 1) the star rating of the review (on a scale of $1-5$ ) } & $\begin{array}{l}{[2,5,10,13,15,18,23,24,25,} \\
26,27,28,29,30,31,32,33, \\
34,35,36,37,38]\end{array}$ \\
\hline & & & \begin{tabular}{|l}
{$\left[\begin{array}{l}6,8,39,40,41,42,43,44,45 \\
46,47,48,49,50,51,52,53]\end{array}\right.$} \\
\end{tabular} \\
\hline & & & $\times[12]$ \\
\hline & Rating $^{2}$ & 1) the quadratic term of the rating & \begin{tabular}{|l|l}
+ & {$[1,2,6,9,34,36,42,46,52,54]$} \\
$-[10,13,31,33]$
\end{tabular} \\
\hline & Review length & $\begin{array}{l}\text { 1) the number of words (word count) in a review } \\
\text { 2) the number of typed characters (character count) in a review }\end{array}$ & $+\begin{array}{l}{[1,2,5,6,7,9,10,12,15,17} \\
18,24,25,26,27,29,30,32 \\
33,34,36,38,39,40,41,42 \\
43,44,45,46,47,48,49,50\end{array}$ \\
\hline
\end{tabular}




\begin{tabular}{|c|c|c|c|c|}
\hline & & & & $\begin{array}{l}52,53,54,55,56,57,58,59, \\
60,61,62,63,64,65]\end{array}$ \\
\hline & & & & {$[35,37]$} \\
\hline & $\begin{array}{l}\text { Review } \\
\text { extremity/ } \\
\text { equivocality/ }\end{array}$ & $\begin{array}{l}\text { 1) moderate review assigned a dummy variable with a value of } \\
0 \text { or } 1 \text { ( } 1 \text { if the rating is } 3 \text { in the } 5 \text {-star range) } \\
\text { 2) extremely negative review assigned a dummy variable with a } \\
\text { value of } 1 \text { (if the review rating is } 1,0 \text { otherwise) }\end{array}$ & & $\begin{array}{l}{[7,17,23,39,42,49,55,56,57,} \\
58]\end{array}$ \\
\hline & & 3) the deviation of the review rating from the average rating & & {$[1,29,41,59,60]$} \\
\hline & Readability & $\begin{array}{l}\text { 1) Flesch-Kincaid Grade Level (FK) [2], Automated } \\
\text { Readability Index (ARI), Gunning Fog Index (FOG) and }\end{array}$ & & $\begin{array}{l}{[6,7,14,30,34,38,39,46,47} \\
48,50,51,52,54,61,64]\end{array}$ \\
\hline & & $\begin{array}{l}\text { Coleman-Liau Index (CLI), indicating reading difficulty [2] } \\
\text { 2) whether the review contains spelling errors or not }\end{array}$ & $x$ & {$[2]$} \\
\hline & Review age & 1) days passed since a given review was published & & $\begin{array}{l}{[5,7,8,13,34,38,39,43,47} \\
48,49,50,51,53,54,55,56 \\
60,65]\end{array}$ \\
\hline & & & & {$[35,36,37,58]$} \\
\hline & & & $x$ & [42] \\
\hline & & $\begin{array}{l}\text { 1) review valence ranges from negative to positive (quantified } \\
\text { range }[-1,+1],[1,3] \text {, or }[1,5] \text { or is assigned a dummy variable }\end{array}$ & & $\begin{array}{l}{[1,10,11,24,28,30,35,54,56,} \\
63,64,66]\end{array}$ \\
\hline & sentiment/ & indicating negative vs positive) & - & {$[36,37,48]$} \\
\hline & polarity & $\begin{array}{l}\text { 2) polarity, sentiment scores of all the words in a review } \\
\text { 3) proportion of positive or/and negative emotional words } \\
\text { within a review }\end{array}$ & $x$ & {$[59,60]$} \\
\hline & |Total votes & 1) total number of votes that a review received & & {$[1,14,15]$} \\
\hline & 10tal votes & & & {$[2,9,18,67]$} \\
\hline & & $\begin{array}{l}\text { 1) review framing/structure/reliability/concreteness/ } \\
\text { informativeness/objective/subjectivity. }\end{array}$ & & $\begin{array}{l}{[8,14,15,31,36,45,47,50,51,} \\
64,68,69]\end{array}$ \\
\hline & Linguistic cues & 2) word usage, functional, explanatory words, etc. & - & {$[49,70]$} \\
\hline & & $\begin{array}{l}\text { 3) language style matching (LSM) of a review } \\
\text { 4) narrative/language/communication style }\end{array}$ & $x$ & {$[13,56,65,67,71]$} \\
\hline & Sidedness & 1) a dummy variable equal to 0 for a one-sided review and 1 for & + & {$[72]$} \\
\hline & 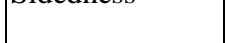 & $\begin{array}{l}\text { two-sided review } \\
\text { tw }\end{array}$ & & {$[56,66]$} \\
\hline & & & & {$[16,17]$} \\
\hline & & 1) discrete emotions & + & {$[30,73]$} \\
\hline & & 2) emotional arousal, which is the average arousal score of & - & {$[56]$} \\
\hline & Emotion & $\begin{array}{l}\text { 1dentified words, ranging from } 1 \text { to } 3 \\
3 \text { ) intensity of emotional expressions, ranging from }-1 \text { to }+1 \\
\text { 4) emoticons, which are graphic demonstrations of facial } \\
\text { expressions, taking values of } 1 \text { (absence) and } 2 \text { (presence) }\end{array}$ & & {$[6,18,33,37,38,65,74]$} \\
\hline & Review picture & $\begin{array}{l}\text { 1) number of pictures in review } \\
\text { 2) picture embedded, a binary variable equal to } 1 \text { if a review } \\
\text { contains embedded picture(s), } 0 \text { otherwise }\end{array}$ & + & {$[8,35,53,54]$} \\
\hline & Total review & 1) total number of available reviews of a product or service & & {$[32,56,63,65]$} \\
\hline & count & 2) popularity & & {$[1,5,7,30,34,38,39,53,58]$} \\
\hline$+\overline{0}$ & & 1) a dummy variable of 0 for search/utilitarian goods and 1 for & & {$[17,28,31,62,74]$} \\
\hline 串胥 & Product type & experience goods/services & & {$[1,5,9,11,15,33]$} \\
\hline D & & $\begin{array}{l}\text { 2) utilitarian value, which is average utilitarian value of the app } \\
\text { category, ranging from }-8 \text { to } 8\end{array}$ & $x$ & [30] \\
\hline & \begin{tabular}{|l|}
$\begin{array}{l}\text { Information } \\
\text { disclosure }\end{array}$ \\
\end{tabular} & $\begin{array}{l}\text { 1) a dummy variable equal to } 1 \text { if a reviewer discloses real } \\
\text { name, location, or profile photo }\end{array}$ & & $\begin{array}{l}{[8,14,15,23,24,25,28,29,35,} \\
43,44,46,52,54,61,62]\end{array}$ \\
\hline & \begin{tabular}{|l|} 
Reviewer \\
experience
\end{tabular} & 1) the number of reviews and/or photos posted by a reviewer & + & $\begin{array}{l}{[28,43,44,51,52,54,55,56,} \\
61,63,72]\end{array}$ \\
\hline 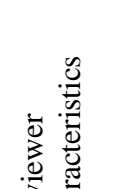 & $\begin{array}{l}\text { Reviewer } \\
\text { expertise }\end{array}$ & $\begin{array}{l}\text { 1) a dummy variable indicating whether a reviewer has top rank } \\
10,000 \text { label or "elite" badge (yes=1, no=0) or reviewer } \\
\text { reputation (reputed=1, novice=0) } \\
\text { 2) number of "elite" badges, credibility of reviewer, rank of a } \\
\text { reviewer, or helpful votes received by a reviewer }\end{array}$ & & $\begin{array}{l}{[1,13,17,26,27,31,32,35,37} \\
39,40,43,44,46,47,48,50 \\
52,53,54,57,59,62,64,68]\end{array}$ \\
\hline 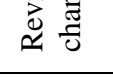 & $\begin{array}{l}\text { Reviewer online } \\
\text { attractiveness }\end{array}$ & $\begin{array}{l}\text { 1) reviewer network centrality, or social ties, which is equal to } \\
\text { the number of friends of a reviewer }\end{array}$ & + & {$[25,39,43,44,47]$} \\
\hline
\end{tabular}


The studies also achieved a consensus on the effects of reviewer characteristics on OCR helpfulness. In particular, 39 studies that investigated reviewer characteristics, including information disclosure, reviewer experience, reviewer expertise, or reviewer online attractiveness, show that each of these factors plays a positive role in enhancing the perceived helpfulness of OCRs (see Table 1).

\subsubsection{Review/product characteristics}

- Review length is defined as the number of words or typed characters in an individual review. It is found that longer reviews positively correlated with the helpfulness of OCRs, with only two outliers yielding opposite results $[35,37]$.

- Readability indicates the degree to which a piece of text is easy for the reader to comprehend. Readability tests were employed to calculate this characteristic, including the FK, ARI, FOG, and CLI tests [2] (see Table 1). These tests approximate the U.S. grade level at which students would comprehend a particular text on a scale of 1 to 12 , except for the FK, a reversed reading ease index that 0 is least readable and 100 most readable. The vast majority of research taking readability into account found that readable reviews helped consumers to understand the review information and were thus more likely to be voted as helpful. Nonetheless, Korfiatis et al. [2] considered all four readability measures in one model and reported mixed findings.

- Review picture is operationalized as whether any picture(s), or the number of picture(s), are embedded in a review. Generally, all of the existing studies considered review picture found that a review accompanied by pictures tends to receive more helpfulness votes.

\subsubsection{Reviewer characteristics}

- Information disclosure is measured as a binary variable. A value of 1 means the reviewer disclosed his or her personal information (e.g., real name, location and/or profile photo) to the public. It has been found that information disclosure is positively related with OCR helpfulness.

- Reviewer experience is the experience of a reviewer with posting reviews. Specifically, it is represented by the numbers of reviews and/or photos posted by a given reviewer. Previous studies have found that reviewer experience positively relates with the perceived helpfulness of reviews.

- Reviewer expertise is indicated by platform-awarded elite badges or rank labels for reviewers' contributions on these platforms. It is possible that reviewers with such badges or labels gain more trust and helpfulness votes for their reviews. Researchers have found that reviewer expertise is positively associated with OCR helpfulness.

- Reviewer online attractiveness is defined as the number of friends or social ties of a reviewer reflected as his or her popularity or attractiveness on online social networks. Previous studies have found that, if a reviewer has more friends, his or her reviews are more likely to be voted as helpful.

\subsection{Inconsistent Findings}

We did observe conflicting findings in regard to the influence of the rest of precursors to OCR helpfulness, including star rating and its quadratic term, review extremity, review age, review valence, review sidedness, and so forth.

Review rating indicates a reviewer's overall assessment of a product or service [5], which is typically measured with a numerical star rating on a scale of one to five stars. Forty studies in our dataset investigated the impact of review rating on OCR helpfulness, yielding contradictory results (see Table 1). Over half of the studies reported a positive impact of review rating on OCR helpfulness, indicating that the higher the positive rating, the more helpful it is considered to be. Meanwhile, 17 studies revealed a negative effect, claiming that negative OCRs are likely to receive higher helpfulness appraisal. Similarly, Zhang et al. [12] reported mixed findings that review ratings affect the perceived helpfulness differently depending on review readers' consumption goals. Some researchers included a quadratic specification of the review rating to account for the nonlinear relationship between review rating and helpfulness [9]. Nonetheless, their findings are inconsistent as well.

Review extremity, which has also been conceptualized as "review equivocality" or "review ambiguity" [23], is often measured using dummy variables, or by assigning different binary values to moderate or equivocal reviews expressing mixed opinions and to extreme or unequivocal reviews. Other researchers have taken a relativism approach and operationalized this concept as "review inconsistency" $[1,39,42,55,60]$, measuring review extremity by the deviation of the star rating of review from the average rating. Yet, it is still unclear whether review extremity enhances or degrades the perceived helpfulness of OCRs (see Table 1). Some studies have reported that extreme reviews are perceived as more helpful than moderate ones inasmuch as they convey a clear message, expediting decision making. However, from the perspective of information diagnosticity, some scholars have argued that a moderate review containing mixed attitudes often provides more balanced evaluations of a product or service. Customers may 
perceive such a review as more helpful because of the inclusion of more diagnostic information.

Review valence [24], indicating the evaluative direction of a review, has also been coined as review sentiment [1, 10] or polarity [37]. Researchers operationalized this factor differently in the existing literature. Some performed content analysis to measure the valence of reviews [24], while others calculated valence through NLP $[6,30]$. Last but not least, several others measured review sentiment directly based on the fraction of positive or negative words [1, 10, 60]. As shown in Table 1, different operationalizations have resulted in inconsistent findings. On the one hand, twothirds of the studies in our sample identified that positive sentiments in OCR texts have a positive impact on perceived helpfulness. On the other hand, some reported a negativity bias effect, with texts embedding negative sentiments being shown as more likely to be perceived as more helpful [36, 37, 48].

Review sidedness was usually derived through content analysis. Researchers used a dummy variable to code reviews as one-sided (containing either pros or cons) or two-sided (mentioning both pros and cons). Mixed findings are reported (see Table 1). For instance, Filieri et al. [72] argued that two-sided reviews had a positive effect on perceived helpfulness, whereas other researchers warned of a negative impact [56, 66]. Schlosser [16] reported that the effect of review sidedness on helpfulness depends on the perceived consistency between the review content and ratings. Chen [17] identified the interplay effects of review sidedness, reviewer expertise, and product type on OCR helpfulness.

Total review votes were collected directly from online data sources as the number of votes cast on a given review. Many researchers also calculated the proportion of helpful votes to total votes. Seven studies in our sample investigated the effect of this variable on OCR helpfulness. Even though the studies including total votes in their models used a uniform means of measurement, antagonistic results are reported (see Table 1). Three studies reported a positive effect of total votes on OCR helpfulness $[1,14,15]$, whereas another four reported a negative effect [2, 9, 18, 67].

Review age is operationalized as the time interval between OCR posting and data collection. The majority of existing research found that review age, or reviews that have been posted for a longer time, are more likely to have more helpful votes. However, four studies indicated a negative effect of review age. $\mathrm{Wu}$ [41] reported mixed findings regarding the effect of review age on OCR helpfulness.

Total review count is defined as the total number of OCRs commenting on a specific brand, service, or product. Most studies found that products with a larger number of OCRs tend to receive less helpfulness votes. However. several recent works reported opposite findings $[32,56,63,65]$. In more recent studies, review age and total review count have been taken into account as control variables.

\subsection{Explanations for Inconsistent Findings}

In this section, we explore possible reasons for the inconsistent findings in the existing studies. Differences in product type may be one reason why mixed or conflicting results appear in regard to the effect of review rating and its quadratic term, review extremity, and review valence. Generally speaking, empirical findings indicate the existence of a product-type effect, affirming that reviews for experience goods are perceived as less helpful than those for search goods [1, $5,9,15]$. Among the twelve studies accounting for the effect of product type, Mudambi and Schuff [9] argued that product type, namely search goods or experience goods, plays an important role in understanding which factors contribute to OCR helpfulness. They reported that product type moderates the effect of review extremity and perceived helpfulness. Pan and Zhang [5] found product type to moderate the relationship between review valence and OCR helpfulness. Thus, failing to take product type into consideration may confound the results.

Another explanation for the mixed findings may be disparities in the measurement and analysis of the contributors. Most studies employ the fraction of helpful votes to total votes as a dependent variable to represent the helpfulness of reviews [1, 2, 6, 9, 10, 15, 18, 23, 24], whereas some studies use the count of helpful votes to measure OCR helpfulness directly [39, 42, 55]. Other researchers in experimental studies have used a dichotomous variable to represent whether an OCR is helpful or not. Additional discrepancies can be observed in the operationalization of other variables, including review extremity, readability, and review valence.

Finally, yet importantly, the lack of consistent findings may also be due to the varied research settings, selection bias, or misuse of statistical analysis. As Mudambi and Schuff [9] pointed out, researchers can only collect the number of total votes and helpful votes, rather than the number of people who read the reviews. It is highly possible that selection bias is inherent in this type of dataset. Some research has suggested employing Heckman's sample selection model [75] to treat this potential bias. Also, studies using Tobit regression or ordinary linear regression models are exposed to this risk. The misuse of statistical tests was also spotted in our sample; for example, when adding an interaction to a model, both the interaction effect and the main effects of each variable alone should be included [76]. Thus, in 
the analysis suffering from the omitted variable bias, the effect of excluded variables is attributed to the included variables, resulting in a biased coefficient estimate. Researchers must be aware of these issues in order to generate unbiased and consistent results.

\subsection{Emerging Trends}

Based on our review, we found that researchers acknowledge that emotions are expressed in OCRs and that these embedded emotions, without a doubt, have an impact on their perceived helpfulness. Among the studies on emotions, most of them simply mapped emotions into two classes, positive and negative. Strictly speaking, measuring emotions in this manner should be termed as review valence or review sentiment rather than emotion. Furthermore, this valence-based approach to measure emotions has been increasingly challenged for its inability to capture the nuance between emotions that differ little in terms of valence $[6,77]$. According to Plutchik's theory on emotion dimensionalities, emotions are classified into eight discrete emotion dimensions [78]. In particular, the discrete emotions of joy, anticipation, surprise, and trust are categorized as positive, whereas angry, anxiety, disgust, and sadness are considered negative. Two articles documented the effects of discrete emotions on perceived helpfulness and demonstrated the necessity of examining discrete emotions in OCR helpfulness literature [6, 18]. Yin et al. [6] demonstrated the effects of two distinct negative emotions, anxiety and anger, on OCR helpfulness. Moreover, anxiety shows a stronger influence than anger, even though they are both negative emotions [6]. Ahmad et al. [18] drew on cognitive appraisal theory to examine the influence of four discrete emotions on perceived helpfulness: hope, anxiety, happiness, and disgust. They confirmed the differential effects of discrete emotions on OCR helpfulness. Nevertheless, academic research on the effects of discrete emotions is far from conclusive.

With the advancement of NLP and text mining techniques, linguistic cues have become popular and widely studied drivers of OCR helpfulness. Researchers not only can identify objective and subjective words [13, 15], functional words [56], and so forth from huge volumes of textual content [47, 48, 49], but can also efficiently assess text similarity [36], LSM [50], review reliability and informativeness [31], and content concreteness [69] in order to explore their effects on the helpfulness of OCRs. Moreover, some researchers have pointed out that review readers prefer clear and structured OCRs [64] to negatively framed ones [45]. Others recommended reviewers to comment on specific product or service attributes when writing OCRs [8, 71]. Nonetheless, research efforts are still necessary to fully uncover the different layers of meaning hidden in OCRs given the richness and versatility of language.

\section{Discussion and Implications}

This paper aimed to facilitate a better understanding on the contributors to OCR helpfulness by providing a comprehensive review of the existing research. Our interdisciplinary and systematic literature review identified 68 high-quality academic papers centering on OCR helpfulness. We examined these articles in regard to their research method, studied factors, operationalization of the contributors, and findings. Based on a content analysis, we extracted and consolidated the contributing factors and categorized them into three categories: review, product, and reviewer characteristics. For each contributor, we outlined its description, operationalization, and corresponding findings. Our results suggest that the findings are consistent with respect to the effects of reviewer characteristics (information disclosure, reviewer experience, reviewer expertise, and reviewer online attractiveness) and three review characteristics (review length, readability, and review pictures). However, the findings are equivocal with respect to the impact of other review and product characteristics (star ratings and its quadratic term, review extremity, review valence, review age, total votes, sidedness, emotions, linguistic cues, and total review count and product type).

This systematic literature review has both theoretical and practical implications. First, it contributes to the existing literature by unraveling the status quo of research on OCR helpfulness and identifying potential research opportunities. Second, this work contributes to the growing literature on OCRs by creating an integrated theoretical framework to facilitate the understanding of consumers' perception of OCR helpfulness. Third, a set of possible reasons for conflicting findings is pinpointed, including the moderation effect of product type, disparities in operationalization, different research settings, selection bias, and misuse of statistical analysis, which help to shed light on the equivocal findings. Researchers may take these factors or issues into consideration in order to generate findings that are more consistent.

The findings derived from our literature review also have practical implications. We have summarized the effects of the contributors to OCR helpfulness and presented them in a way that is more accessible to practitioners. First, it can help OCR platforms understand how to refine their appraisal mechanism of OCR helpfulness and to prioritize reviews that are more helpful to reduce information overload and facilitate consumers' decision making; this, in turn, can increase the popularity of the platform. Second, the findings can 
serve as guidelines for companies to identify potentially popular OCRs in a timelier manner, which can facilitate them in taking corrective action against potentially harmful OCRs and promotive action toward favorable ones. Third, by promptly discerning potentially helpful OCRs from misleading ones, consumers can make better decisions, thereby leading to post-purchase satisfaction. Finally, major contributors to OCR helpfulness are also important for reviewers in order to understand how to write helpful reviews. In this digital age, such information is important for all the stakeholders in the online market.

\section{Agenda for Future Research}

Adhering to the objectives of this systematic literature review, we also offer recommendations for future research in this field. Based on our results, a number of fruitful research directions can by identified for future works.

First, our literature analysis highlights a paucity of intensive exploration of the impacts of discrete emotions and linguistic cues on review helpfulness. Guided by theories in the psychological domain, plenty of widely accepted emotion dictionaries are available for extracting emotions in texts. Machine learning is also an apropos technique for this task. Researchers following this agenda can use the aforementioned approaches to detect discrete emotions and thereby study their differential effects on perceived helpfulness. Furthermore, the existing literature studied review sidedness as a dichotomous variable, similarly neglecting the subtleties of emotion. Future research could aim to quantify the level of sidedness, as it is critical to understand these nuances in order to truly comprehend OCRs. Additionally, it is worth further investigating the linguistic features of reviews, such as argument structure and language style, as these might interact with emotions and sidedness and affect OCR helpfulness.

Second, the results show that the literature on the drivers of OCR helpfulness is rather fragmented. Basic review characteristics, such as rating, review extremity and valance, review age, and so forth, were the most investigated, albeit controversial, factors. Other potentially influential factors were only analyzed sporadically. Future research can explore theories from different disciplines and to use them to propose integrative models. For example, researchers can further investigate how other factors such as purchase context, price, risk, first-time versus repeat buying, consumer skepticism, etc., affect OCR evaluation.

Finally, the current studies have extensively investigated the contributors to OCR helpfulness based on secondary data. Meanwhile, the effects of the design and functionality of OCR systems remain insufficiently investigated, for instance, the roles of big data analytics and information curation in supporting consumers' evaluation and appreciation of review information. We therefore encourage researchers to adopt experimental designs to study how the IT artifacts of OCR platforms incentivize the creation or facilitate the consumption of helpful reviews in addition to how these artifacts influence customers' decision making.

\section{References}

[1] H. Baek, J. Ahn, and Y. Choi, "Helpfulness of Online Consumer Reviews: Readers' Objectives and Review Cues", International Journal of Electronic Commerce 17(2), 2012, pp. 99-126.

[2] N. Korfiatis, E. García-Bariocanal, and S. Sánchez-Alonso, "Evaluating Content Quality and Helpfulness of Online Product Reviews: The Interplay of Review Helpfulness vs. Review Content", Electronic Commerce Research and Applications 11(3), 2012, pp. 205-217.

[3] Podium, 2017 State of Online Reviews, 2017.

[4] W. Ye, R. Law, B. Gu, and W. Chen, "The Influence of User-Generated Content on Traveler Behavior: An Empirical Investigation on the Effects of E-Word-of-Mouth to Hotel Online Bookings", Computers in Human Behavior 27, 2011, pp. 634-639.

[5] Y. Pan and J.Q. Zhang, "Born Unequal: A Study of the Helpfulness of User-Generated Product Reviews", Journal of Retailing 87(4), 2011, pp. 598-612.

[6] D. Yin, S.D. Bond, and H. Zhang, "Anxious or Angry? Effects of Discrete Emotions on the Perceived Helpfulness of Online Reviews", MIS Quarterly 38(2), 2014, pp. 539-560.

[7] B. Fang, Q. Ye, D. Kucukusta, and R. Law, "Analysis of the Perceived Value of Online Tourism Reviews: Influence of Readability and Reviewer Characteristics", Tourism Management 52, 2016, pp. 498-506.

[8] X. Sun, M. Han, and J. Feng, "Helpfulness of Online Reviews: Examining Review Informativeness and Classification Thresholds by Search Products and Experience Products”, Decision Support Systems 124, 2019, pp. 113099.

[9] S.M. Mudambi and D. Schuff, "What Makes a Helpful Online Review? A Study of Customer Reviews on Amazon.com", MIS Quarterly 34(1), 2010, pp. 185-200.

[10] R. Ullah, A. Zeb, and W. Kim, "The Impact of Emotions on the Helpfulness of Movie Reviews", Journal of Applied Research and Technology 13(3), 2015, pp. 359-363.

[11] S. Sen and D. Lerman, "Why Are You Telling Me This? An Examination into Negative Consumer Reviews on the Web", Journal of Interactive Marketing 21(4), 2007, pp. 7694.

[12] J.Q. Zhang, G. Craciun, and D. Shin, "When Does Electronic Word-of-Mouth Matter? A Study of Consumer Product Reviews", Journal of Business Research 63(12), 2010, pp. 1336-1341.

[13] M. Scholz and V. Dorner, "The Recipe for the Perfect Review? An Investigation into the Determinants of Review Helpfulness", Business and Information Systems Engineering 5(3), 2013, pp. 141-151.

[14] A. Ghose and P.G. Ipeirotis, "Estimating the Helpfulness 
and Economic Impact of Product Reviews: Mining Text and Reviewer Characteristics", IEEE Transactions on Knowledge and Data Engineering 23(10), 2011, pp. 1498-1512.

[15] B. Einar, L.J. Havro, and O. Moen, "An Empirical Investigation of Self-Selection Bias and Factors Influencing Review Helpfulness", International Journal of Business and Management 10(7), 2015, pp. 16-31.

[16] A.E. Schlosser, "Can including pros and cons increase the helpfulness and persuasiveness of online reviews? The interactive effects of ratings and arguments", Journal of Consumer Psychology 21(3), 2011, pp. 226-239.

[17] M.Y. Chen, "Can Two-Sided Messages Increase the Helpfulness of Online Reviews?", Online Information Review 40(3), 2016, pp. 316-332.

[18] S.N. Ahmad and M. Laroche, "How Do Expressed Emotions Affect the Helpfulness of a Product Review? Evidence from Reviews Using Latent Semantic Analysis", International Journal of Electronic Commerce 20(1), 2015, pp. 76-111.

[19] A. Oshin, P.R. Srivastava, and A. Rakshit, "Assessment, Implication, and Analysis of Online Consumer Reviews: A Literature Review", Pacific Asia Journal of the Association for Information Systems 9(2), 2017, pp. 43-74.

[20] H. Hong, D. Xu, G.A. Wang, and W. Fan, "Understanding the Determinants of Online Review Helpfulness: A Meta-Analytic Investigation", Decision Support Systems 102, 2017, pp. 1-11.

[21] J. Webster and R.T. Watson, "Analyzing the Past to Prepare for the Future: Writing a Literature Review", MIS Quarterly 26(2), 2002, pp. xiii-xxiii.

[22] D. Buhalis and R. Law, "Progress in Information Technology and Tourism Management: 20 Years on and 10 Years after the Internet-The State of eTourism Research", Tourism Management 29(4), 2008, pp. 609-623.

[23] C. Forman, A. Ghose, and B. Wiesenfeld, "Examining the Relationship Between Reviews and Sales: The Role of Reviewer Identity Disclosure in Electronic Markets", Information Systems Research 19(3), 2008, pp. 291-313.

[24] L.M. Willemsen, P.C. Neijens, F. Bronner, and J.A. de Ridder, “'Highly Recommended!' The Content Characteristics and Perceived Usefulness of Online Consumer Reviews", Journal of Computer-Mediated Communication 17(1), 2011, pp. 19-38.

[25] Z. Liu and S. Park, "What Makes a Useful Online Review? Implication for Travel Product Websites", Tourism Management 47, 2015, pp. 140-151.

[26] S. Quaschning, M. Pandelaere, and I. Vermeir, "When Consistency Matters: The Effect of Valence Consistency on Review Helpfulness", Journal of Computer-Mediated Communication 20(2), 2015, pp. 136-152.

[27] A.H. Huang, K. Chen, D.C. Yen, and T.P. Tran, "A Study of Factors that Contribute to Online Review Helpfulness", Computers in Human Behavior 48, 2015, pp. 17-27.

[28] A. Agnihotri and S. Bhattacharya, "Online Review Helpfulness: Role of Qualitative Factors", Psychology and Marketing 33(11), 2016, pp. 1006-1017.

[29] S. Karimi and F. Wang, "Online Review Helpfulness: Impact of Reviewer Profile Image", Decision Support Systems 96, 2017, pp. 39-48.

[30] D. Yin, S.D. Bond, and H. Zhang, "Keep Your Cool or Let It Out: Nonlinear Effects of Expressed Arousal on
Perceptions of Consumer Reviews", Journal of Marketing Research 54(3), 2017, pp. 447-463.

[31] A.Y.K. Chua and S. Banerjee, "Analyzing Review Efficacy on Amazon.com: Does the Rich Grow Richer?", Computers in Human Behavior 75, 2017, pp. 501-509.

[32] S. Lu, J. Wu, and S.-L. (Allen) Tseng, "How Online Reviews Become Helpful: A Dynamic Perspective", Journal of Interactive Marketing 44, 2018, pp. 17-28.

[33] G. Ren and T. Hong, "Examining the Relationship Between Specific Negative Emotions and the Perceived Helpfulness of Online Reviews", Information Processing and Management 56(4), 2019, pp. 1425-1438.

[34] Y. Wang, J. Wang, and T. Yao, "What Makes a Helpful Online Review? A Meta-Analysis of Review Characteristics", Electronic Commerce Research 19(2), 2019, pp. 257-284.

[35] S. Yang, Y. Zhou, J. Yao, Y. Chen, and J. Wei, "Understanding Online Review Helpfulness in Omnichannel Retailing", Industrial Management and Data Systems 119(8), 2019, pp. 1565-1580.

[36] Y. Zhou, S. Yang, Y. Li, Y. Chen, J. Yao, and A. Qazi, "Does the Review Deserve more Helpfulness when its Title Resembles the Content? Locating Helpful Reviews by Text Mining", Information Processing and Management 57(2), 2020.

[37] S. Chatterjee, "Drivers of Helpfulness of Online Hotel Reviews: A Sentiment and Emotion Mining Approach", International Journal of Hospitality Management 85, 2020, pp. 102356.

[38] G. Craciun, W. Zhou, and Z. Shan, "Discrete Emotions Effects on Electronic Word-of-Mouth Helpfulness: The Moderating Role of Reviewer Gender and Contextual Emotional Tone", Decision Support Systems 130, 2020, pp. 113226.

[39] L. Zhu, G. Yin, and W. He, "Is This Opinion Leader's Review Useful? Peripheral Cues for Online Review Helpfulness", Journal of Electronic Commerce Research 15(4), 2014, pp. 267-280.

[40] A.Y.K. Chua and S. Banerjee, "Understanding Review Helpfulness as a Function of Reviewer Reputation, Review Rating, and Review Depth", Journal of the Association for Information Science and Technology 66(2), 2015, pp. 354-362. [41] D. Yin, S. Mitra, and H. Zhang, "When Do Consumers Value Positive vs. Negative Reviews? An Empirical Investigation of Confirmation Bias in Online Word of Mouth", Information Systems Research 27(1), 2016, pp. 131-144.

[42] J. Wu, "Review Popularity and Review Helpfulness: A Model for User Review Effectiveness", Decision Support Systems 97, 2017, pp. 92-103.

[43] B. Guo and S. Zhou, "What Makes Population Perception of Review Helpfulness: An Information Processing Perspective", Electronic Commerce Research 17(4), 2017, pp. 585-608.

[44] S. Zhou and B. Guo, "The Order Effect on Online Review Helpfulness: A Social Influence Perspective", Decision Support Systems 93, 2017, pp. 77-87.

[45] S.P. Eslami, M. Ghasemaghaei, and K. Hassanein, "Which Online Reviews Do Consumers Find Most Helpful? A Multi-Method Investigation", Decision Support Systems 113, 2018, pp. 32-42.

[46] R. Filieri, E. Raguseo, and C. Vitari, "When Are Extreme Ratings More Helpful? Empirical Evidence on the Moderating 
Effects of Review Characteristics and Product Type", Computers in Human Behavior 88, 2018, pp. 134-142.

[47] H. Li, C. (Renee) Wang, F. Meng, and Z. Zhang, "Making Restaurant Reviews Useful and/or Enjoyable? The Impacts of Temporal, Explanatory, and Sensory Cues", International Journal of Hospitality Management 83, 2019, pp. 257-265.

[48] Y. Zhou and S. Yang, "Roles of Review Numerical and Textual Characteristics on Review Helpfulness Across Three Different Types of Reviews", IEEE Access 7, 2019, 2776927780.

[49] F. Wang and S. Karimi, "This Product Works Well (for $\mathrm{Me}$ ): The Impact of First-Person Singular Pronouns on Online Review Helpfulness", Journal of Business Research 104, 2019, pp. 283-294.

[50] A.X. Liu, Y. Xie, and J. Zhang, "It's Not Just What You Say, but How You Say It: The Effect of Language Style Matching on Perceived Quality of Consumer Reviews", Journal of Interactive Marketing 46, 2019.

[51] X. Wang, L. (Rebecca) Tang, and E. Kim, "More than Words: Do Emotional Content and Linguistic Style Matching Matter on Restaurant Review Helpfulness?", International Journal of Hospitality Management 77, 2019, pp. 438-447.

[52] S. Liang, M. Schuckert, and R. Law, "How to Improve the Stated Helpfulness of Hotel Reviews? A Multilevel Approach", International Journal of Contemporary Hospitality Management 31(2), 2019, pp. 953-977.

[53] L. Fan and X. Zhang, "The Combination Signaling Effect of Text and Image on Mobile Phone Review Helpfulness - The Moderating Effect of Signaling Environment", IEEE Access 8, 2020, pp. 122736-122746.

[54] V. Srivastava and A.D. Kalro, "Enhancing the Helpfulness of Online Consumer Reviews: The Role of Latent (Content) Factors", Journal of Interactive Marketing 48, 2019, pp. 33-50.

[55] B. Gao, N. Hu, and I. Bose, "Follow the Herd or Be Myself? An Analysis of Consistency in Behavior of Reviewers and Helpfulness of Their Reviews", Decision Support Systems 95, 2017, pp. 1-11.

[56] A. März, S. Schubach, and J.H. Schumann, "“Why Would I Read a Mobile Review?' Device Compatibility Perceptions and Effects on Perceived Helpfulness", Psychology \& Marketing 34(2), 2017, pp. 119-137.

[57] R. Filieri, E. Raguseo, and C. Vitari, "What Moderates the Influence of Extremely Negative Ratings? The Role of Review and Reviewer Characteristics", International Journal of Hospitality Management 77, 2019, pp. 333-341.

[58] Y. Zhu, M. Liu, X. Zeng, and P. Huang, "The Effects of Prior Reviews on Perceived Review Helpfulness: A Configuration Perspective", Journal of Business Research 110, 2020, pp. 484-494.

[59] K.K.Y. Kuan, K.-L. Hui, P. Prasarnphanich, and H.-Y. Lai, "What Makes a Review Voted? An Empirical Investigation of Review Voting in Online Review Systems", Journal of the Association for Information Systems 16(1), 2015, pp. 48-71.

[60] Y. Hong, N. Huang, G. Burtch, and C. Li, "Culture, Conformity, and Emotional Suppression in Online Reviews", Journal of the Association for Information Systems 17(11), 2016, pp. 737-758.

[61] S. Park and J.L. Nicolau, "Asymmetric Effects of Online Consumer Reviews", Annals of Tourism Research 50, 2015, pp. 67-83.

[62] S. Lee and J.Y. Choeh, "The Determinants of Helpfulness of Online Reviews", Behaviour \& Information Technology 35(10), 2016, pp. 853-863.

[63] M. Lee, M. Jeong, and J. Lee, "Roles of Negative Emotions in Customers' Perceived Helpfulness of Hotel Reviews on a User-Generated Review Website: A Text Mining Approach", International Journal of Contemporary Hospitality Management 29(2), 2017, pp. 762-783.

[64] H. Risselada, L. de Vries, and M. Verstappen, "The Impact of Social Influence on the Perceived Helpfulness of Online Consumer Reviews", European Journal of Marketing 52(3-4), 2018, pp. 619-636.

[65] G.H. Huang, C.T. Chang, A. Bilgihan, and F. Okumus, "Helpful or Harmful? A Double-Edged Sword of Emoticons in Online Review Helpfulness", Tourism Management 81, 2020.

[66] I. Pentina, A.A. Bailey, and L. Zhang, "Exploring Effects of Source Similarity, Message Valence, and Receiver Regulatory Focus on Yelp Review Persuasiveness and Purchase Intentions", Journal of Marketing Communications 24(2), 2018, pp. 125-145.

[67] A. Qazi, K.B. Shah Syed, R.G. Raj, E. Cambria, M. Tahir, and D. Alghazzawi, "A Concept-Level Approach to the Analysis of Online Review Helpfulness", Computers in Human Behavior 58, 2016, pp. 75-81.

[68] G. Yin, W. Liu, and S. Zhu, "What Makes a Helpful Online Review? - The Perspective of Information Adoption and Social Network", Library and Information Service 56(16), 2012, pp. 140-147.

[69] L. Huang, C. Hoo Tan, W. Ke, and K.-K. Wei, "Helpfulness of Online Review Content: The Moderating Effects of Temporal and Social Cues", Journal of the Association for Information Systems 19(6), 2018, pp. 503-522. [70] S. Shin, N. Chung, Z. Xiang, and C. Koo, "Assessing the Impact of Textual Content Concreteness on Helpfulness in Online Travel Reviews", Journal of Travel Research 58(4), 2019, pp. 579-593.

[71] F. Hu, "What Makes a Hotel Review Helpful? An Information Requirement Perspective", Journal of Hospitality Marketing \& Management 29(5), 2020, pp. 571-591.

[72] R. Filieri, F. McLeay, B. Tsui, and Z. Lin, "Consumer Perceptions of Information Helpfulness and Determinants of Purchase Intention in Online Consumer Reviews of Services", Information \& Management 55(8), 2018, pp. 956-970.

[73] M.J. Chen, "Examining the Influence of Emotional Expressions in Online Consumer Reviews on Perceived Helpfulness", Information Processing and Management 57(6), 2020, pp. 102266.

[74] E. Ismagilova, Y.K. Dwivedi, and E. Slade, "Perceived Helpfulness of eWOM: Emotions, Fairness and Rationality", Journal of Retailing and Consumer Services 53, 2020.

[75] J.J. Heckman, "Sample Selection Bias as a Specification Error", Econometrica 47(1), 1979, pp. 153-161.

[76] J.M. Wooldridge, Introductory Econometrics: A Modern Approach, Cengage Learning, Mason, OH, 2013.

[77] J.R.J. Fontaine, K.R. Scherer, E.B. Roesch, and P.C. Ellsworth, "The World of Emotions is Not Two-Dimensional", Psychological Science 18(12), 2007, pp. 1050-1057.

[78] R. Plutchik, The Psychology and Biology of Emotion, HarperCollins College Publishers, New York, NY, US, 1994. 